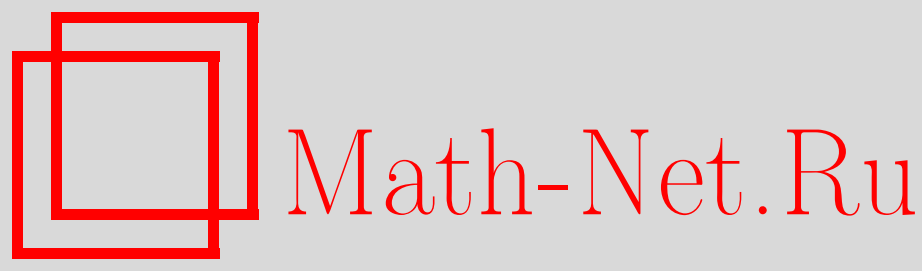

Р. С. Пусев, Асимптотика малых уклонений процессов Боголюбова в квадратичной норме, ТМФ, 2010, том 165, номер 1, 134-144

DOI: https://doi.org/10.4213/tmf6567

Использование Общероссийского математического портала Math-Net.Ru подразумевает, что вы прочитали и согласны с пользовательским соглашением http://www.mathnet.ru/rus/agreement

Параметры загрузки:

IP : 54.147 .182 .235

26 апреля 2023 г., 12:39:40

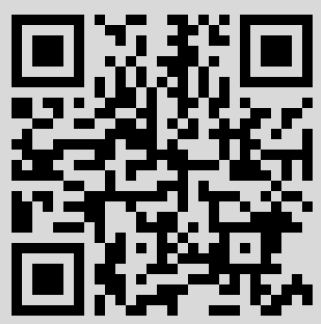




\title{
АСИМПТОТИКА МАЛЫХ УКЛОНЕНИЙ ПРОЦЕССОВ БОГОЛЮБОВА В КВАДРАТИЧНОЙ НОРМЕ
}

\begin{abstract}
Получены результаты о малых уклонениях гауссовской меры Боголюбова, возникающей в теории статистического равновесия квантовых систем. Найдена точная асимптотика вероятностей малых уклонений в гильбертовой норме для некоторых случайных процессов, связанных с процессами Боголюбова.
\end{abstract}

Ключевые слова: мера Боголюбова, малые уклонения.

Мера Боголюбова, введенная в работах [1], [2], играет важную роль в теории статистического равновесия квантовых систем. Она возникает в представлении гиббсовских равновесных средних от бозе-операторов в виде функциональных интегралов с использованием метода $T$-произведений Боголюбова (см. работу [3]).

Определим процесс Боголюбова $\xi(t), t \in[0,1]$, как гауссовский процесс с нулевым средним и ковариационной функцией

$$
\mathrm{E} \xi(t) \xi(s)=\frac{1}{2 \omega \operatorname{sh}(\omega / 2)} \operatorname{ch}\left(\omega|t-s|-\frac{\omega}{2}\right), \quad t, s \in[0,1], \quad \omega>0 .
$$

Из общих результатов теории случайных процессов следует, что траектории процесса Боголюбова почти наверное (п.н.) непрерывны. Из определения (1) получаем $\mathrm{E}(\xi(1)-\xi(0))^{2}=0$, значит, $\xi(0)=\xi(1)$ п. н. Таким образом, почти все траектории процесса Боголюбова принадлежат пространству $C^{0}[0,1]$ непрерывных функций $x(t)$, определенных на отрезке $[0,1]$ и удовлетворяющих условию $x(0)=x(1)$. Мерой Боголюбова $\mu_{\mathrm{B}}$ называется распределение процесса $\xi(t)$ в пространстве $C^{0}[0,1]$, снабженном равномерной метрикой.

Свойства меры Боголюбова, функциональных интегралов по этой мере и траекторий процесса Боголюбова изучались в работах [2], [4], [5]. В статье [2] были построены приближенные формулы для функциональных интегралов по мере Боголюбова, точные для функциональных многочленов. В работе [4] установлено, что подобно винеровскому процессу почти все траектории процесса Боголюбова не удовлетворяют условию Гёльдера с показателем $\gamma>1 / 2$, а значит, являются недифференцируемыми. В статье [5] получены некоторые асимптотические формулы для

* Санкт-Петербургский государственный университет, Санкт-Петербург, Россия. E-mail: Ruslan.Pusev@math.spbu.ru 
функциональных интегралов по мере Боголюбова, а также вычислена важная для многих задач теории вероятностей и математической физики асимптотика вероятностей больших уклонений процессов Боголюбова в $L_{p}$-нормах.

Еще одной фундаментальной характеристикой случайных процессов, используемой, например, при оценке точности приближений случайных процессов и вычислении метрической энтропии различных множеств, является асимптотика их малых уклонений в той или иной норме. Теория малых уклонений для норм гауссовских процессов бурно развивается в последнее десятилетие (см., например, работы [6], [7]). Недавно была установлена связь малых уклонений с задачами математической статистики: функциональным анализом данных и непараметрическим байесовским оцениванием [8]-[10].

В большинстве случаев удается найти грубую (логарифмическую) асимптотику вероятностей малых уклонений гауссовских процессов. Вычисление точной асимптотики является значительно более трудной задачей, решение которой известно лишь для немногих процессов [11]-[16].

В настоящей работе мы вычисляем точную асимптотику вероятностей малых уклонений в $L_{2}$-норме для самого процесса Боголюбова и некоторых процессов, связанных с ним. Для случайного процесса $X(t), t \in[0,1]$, положим

$$
\|X\|=\left(\int_{0}^{1} X^{2}(t) d t\right)^{1 / 2} .
$$

Нас интересует асимптотика при $\varepsilon \rightarrow 0$ вероятности $\mathrm{P}\{\|X\| \leqslant \varepsilon\}$.

В силу классического разложения Карунена-Лоэва (см., например, работу [17]) имеет место равенство по распределению

$$
\|X\|^{2}=\sum_{k=1}^{\infty} \lambda_{k} \eta_{k}^{2}
$$

где $\eta_{k}, k \in \mathbb{N},-$ независимые стандартные гауссовские случайные величины, а числа $\lambda_{k}>0, k \in \mathbb{N}, \sum_{k} \lambda_{k}<\infty$, суть собственные значения интегрального уравнения

$$
\lambda f(t)=\int_{0}^{1} G(t, s) f(s) d s, \quad t \in[0,1],
$$

в котором ядро $G(t, s)$ - это ковариационная функция процесса $X$. Таким образом, исходная задача сводится к описанию поведения при $\varepsilon \rightarrow 0$ вероятности $\mathrm{P}\left\{\sum_{k=1}^{\infty} \lambda_{k} \eta_{k}^{2} \leqslant \varepsilon^{2}\right\}$.

Мы используем следующую лемму, принадлежащую М. А. Лифшицу.

Лемма. Пусть $V_{1}, V_{2}>0$ - независимъе случайные величины с известной асимптотикой вероятностей мальх уклонений: при $r \rightarrow 0$

$$
\mathrm{P}\left\{V_{1} \leqslant r\right\} \sim K_{1} r^{a_{1}} \exp \left(-D_{1}^{d+1} r^{-d}\right), \quad \mathrm{P}\left\{V_{2} \leqslant r\right\} \sim K_{2} r^{a_{2}} \exp \left(-D_{2}^{d+1} r^{-d}\right),
$$

где $a_{1}, a_{2}$ - произвольнье вещественные числа, а $K_{1}, K_{2}, D_{1}, D_{2}, d$ - произвольные положительные числа. Тогда для суммы $V_{1}+V_{2}$ верно соотношение

$$
\mathrm{P}\left\{V_{1}+V_{2} \leqslant r\right\} \sim K r^{a} \exp \left(-D^{d+1} r^{-d}\right),
$$


¿əe

$$
D=D_{1}+D_{2}, \quad a=a_{1}+a_{2}-\frac{d}{2}, \quad K=K_{1} K_{2} \sqrt{\frac{2 \pi d}{d+1}} \cdot \frac{D_{1}^{a_{1}+1 / 2} D_{2}^{a_{2}+1 / 2}}{D^{a+1 / 2}} .
$$

Доказательство леммы аналогично получению формулы (5) ниже.

Рассмотрим задачу о нахождении точной асимптотики малых уклонений для процесса Боголюбова.

Теорема 1. При $\varepsilon \rightarrow 0$ верно соотношение

$$
\mathrm{P}\{\|\xi\| \leqslant \varepsilon\} \equiv \mu_{\mathrm{B}}\left\{x: \int_{0}^{1} x^{2}(t) d t \leqslant \varepsilon^{2}\right\} \sim \frac{4 \sqrt{2} \operatorname{sh}(\omega / 2)}{\sqrt{\pi}} \varepsilon \exp \left(-\frac{1}{8} \varepsilon^{-2}\right) .
$$

ДокАзАтЕЛьство. Собственные числа ковариации равны [2]

$$
\lambda_{k}=\frac{1}{\omega^{2}+(2 \pi k)^{2}}, \quad k \in \mathbb{Z} .
$$

Согласно теореме 1 из работы [12] при $\varepsilon \rightarrow 0$

$$
\mathrm{P}\left\{\sum_{k \geqslant 1}\left(4 \pi^{2} k^{2}+\omega^{2}\right)^{-1} \eta_{k}^{2} \leqslant \varepsilon^{2}\right\} \sim 4 \sqrt{\frac{\operatorname{sh}(\omega / 2)}{\pi \omega}} \exp \left(-\frac{1}{32} \varepsilon^{-2}\right) .
$$

Применение леммы дает

$$
\mathrm{P}\left\{\sum_{k \in \mathbb{Z}, k \neq 0}\left(4 \pi^{2} k^{2}+\omega^{2}\right)^{-1} \eta_{k}^{2} \leqslant \varepsilon^{2}\right\} \sim \frac{4 \operatorname{sh}(\omega / 2)}{\sqrt{2 \pi} \omega} \varepsilon^{-1} \exp \left(-\frac{1}{8} \varepsilon^{-2}\right) .
$$

Асимптотику вероятности $\mathrm{P}\left\{\sum_{k \in \mathbb{Z}}\left(4 \pi^{2} k^{2}+\omega^{2}\right)^{-1} \eta_{k}^{2} \leqslant \varepsilon^{2}\right\}$ вычислим, действуя аналогично работе [13] (см. предложение 6.4 в этой работе). Данная вероятность представляет собой свертку двух функций распределения с известным поведением в нуле. Именно, при $x \rightarrow 0$

$$
\frac{d}{d x} \mathrm{P}\left\{\omega^{-2} \eta_{0}^{2} \leqslant x\right\}=\frac{d}{d x}(2 \Phi(\omega \sqrt{x})-1) \sim \frac{\omega}{\sqrt{2 \pi x}},
$$

значит,

$$
\begin{aligned}
\mathrm{P}\left\{\sum_{k \in \mathbb{Z}}\left(4 \pi^{2} k^{2}+\omega^{2}\right)^{-1} \eta_{k}^{2} \leqslant \varepsilon^{2}\right\} & \\
& \sim \frac{2 \operatorname{sh}(\omega / 2)}{\pi} \int_{0}^{\varepsilon^{2}} x^{-1 / 2}\left(\varepsilon^{2}-x\right)^{-1 / 2} \exp \left(-\frac{1}{8}\left(\varepsilon^{2}-x\right)^{-1}\right) d x= \\
= & \frac{4 \operatorname{sh}(\omega / 2)}{\pi} \int_{0}^{1}\left(1-x^{2}\right)^{-1 / 2} \exp \left(-\frac{1}{8 \varepsilon^{2}}\left(1-x^{2}\right)^{-1}\right) d x
\end{aligned}
$$

Применяя метод Лапласа (см., например, монографию [18]), получаем

$$
\int_{0}^{1}\left(1-x^{2}\right)^{-1 / 2} \exp \left(-\frac{1}{8 \varepsilon^{2}}\left(1-x^{2}\right)^{-1}\right) d x \sim \sqrt{2 \pi} \varepsilon \exp \left(-\frac{1}{8 \varepsilon^{2}}\right) .
$$


Подставляя (4) в (3), имеем

$$
\mathrm{P}\left\{\sum_{k \in \mathbb{Z}}\left(4 \pi^{2} k^{2}+\omega^{2}\right)^{-1} \eta_{k}^{2} \leqslant \varepsilon^{2}\right\} \sim \frac{4 \sqrt{2} \operatorname{sh}(\omega / 2)}{\sqrt{\pi}} \varepsilon \exp \left(-\frac{1}{8 \varepsilon^{2}}\right) .
$$

Теорема доказана.

В следующей теореме получена точная асимптотика вероятностей малых уклонений для процесса Боголюбова с экспоненциальным весом.

Теорема 2. Пусть $q \neq 0$. При $\varepsilon \rightarrow 0$ верно соотношение

$$
\begin{gathered}
\mathrm{P}\left\{\int_{0}^{1} \xi^{2}(t) e^{2 q t} d t \leqslant \varepsilon^{2}\right\} \equiv \mu_{\mathrm{B}}\left\{x: \int_{0}^{1} x^{2}(t) e^{2 q t} d t \leqslant \varepsilon^{2}\right\} \sim \\
\sim \frac{4 \sqrt{2} \operatorname{sh}(\omega / 2) q}{\sqrt{\pi \operatorname{ch}(q / 2)}\left(e^{q}-1\right)} \varepsilon \exp \left(-\frac{\left(e^{q}-1\right)^{2}}{8 q^{2}} \varepsilon^{-2}\right) .
\end{gathered}
$$

ДокАЗАТЕЛЬСтво. Из формулы (3.7) в статье [5] и леммы 2.1 в работе [14] следует, что собственные числа ковариации суть $\lambda_{k}=\mu_{k}^{-1}$, где $\mu_{k}$ - собственные числа краевой задачи

$$
\begin{gathered}
y^{\prime \prime}-\left(\omega^{2}-\mu e^{2 q t}\right) y=0, \quad t \in[0,1], \\
y(0)-y(1)=y^{\prime}(0)-y^{\prime}(1)=0 .
\end{gathered}
$$

Положим $\vartheta=\left(e^{q}-1\right) / q$. С учетом теоремы 1.2 из работы [16] нам достаточно показать, что

$$
C_{\mathrm{dist}}^{2} \equiv \prod_{k=1}^{\infty} \frac{\vartheta^{2} \mu_{k}}{(\pi(k-1 / 2))^{2}}=\frac{2 \operatorname{sh}^{2}(\omega / 2)}{\operatorname{ch}(q / 2)}
$$

Общим решением уравнения (7) является (см. справочник [19])

$$
y(t)=c_{1} J_{|\omega / q|}\left(\frac{\sqrt{\mu}}{q} e^{q t}\right)+c_{2} N_{|\omega / q|}\left(\frac{\sqrt{\mu}}{q} e^{q t}\right)
$$

где $J_{\nu}$ и $N_{\nu}$ - функции Бесселя порядка $\nu$ первого и второго рода соответственно.

Используя граничные условия, получаем, что $\sqrt{\mu_{k}}-$ положительные корни функции

$$
F(\zeta)=\operatorname{det}\left(\begin{array}{cc}
J_{|\omega / q|}(\zeta / q)-J_{|\omega / q|}\left(e^{q} \zeta / q\right) & N_{|\omega / q|}(\zeta / q)-N_{|\omega / q|}\left(e^{q} \zeta / q\right) \\
\zeta J_{|\omega / q|}^{\prime}(\zeta / q)-e^{q} \zeta J_{|\omega / q|}^{\prime}\left(e^{q} \zeta / q\right) & \zeta N_{|\omega / q|}^{\prime}(\zeta / q)-e^{q} \zeta N_{|\omega / q|}^{\prime}\left(e^{q} \zeta / q\right)
\end{array}\right) .
$$

Заметим, что $F(-\zeta)=F(\zeta)$. Поскольку при $\operatorname{Re} \zeta \geqslant 0$ и $|\zeta| \rightarrow \infty$ справедливы соотношения (см., например, [20])

$$
\begin{aligned}
J_{\nu}(\zeta) & =\sqrt{\frac{2}{\pi \zeta}} \cos \left(\zeta-\frac{\pi}{2} \nu-\frac{\pi}{4}\right)\left(1+O\left(|\zeta|^{-1}\right)\right), \\
N_{\nu}(\zeta) & =\sqrt{\frac{2}{\pi \zeta}} \sin \left(\zeta-\frac{\pi}{2} \nu-\frac{\pi}{4}\right)\left(1+O\left(|\zeta|^{-1}\right)\right),
\end{aligned}
$$


прямым вычислением получаем

$$
|F(\zeta)|=\frac{4|q|}{\pi} \cdot\left|\operatorname{ch}\left(\frac{q}{2}\right) \cos (\vartheta \zeta)-1\right|+O\left(|\zeta|^{-1}\right) .
$$

Положим $\Psi(\zeta)=\cos (\vartheta \zeta)$. При $|\zeta| \rightarrow \infty, \arg \zeta \neq \pi j / 2, j \in \mathbb{Z}$, получаем

$$
\frac{|F(\zeta)|}{|\Psi(\zeta)|} \rightarrow \frac{4|q| \operatorname{ch}(q / 2)}{\pi}
$$

По теореме Иенсена (см., например, монографию [21])

$$
C_{\text {dist }}^{2}=\frac{|F(0)|}{|\Psi(0)|} \exp \left(\lim _{\rho \rightarrow \infty} \frac{1}{2 \pi} \int_{0}^{2 \pi} \ln \frac{\left|\Psi\left(\rho e^{i \theta}\right)\right|}{\left|F\left(\rho e^{i \theta}\right)\right|} d \theta\right) .
$$

Подынтегральная функция, очевидно, имеет суммируемую мажоранту, и теорема Лебега дает

$$
C_{\mathrm{dist}}^{2}=\frac{|F(0)|}{|\Psi(0)|} \frac{\pi}{4|q| \operatorname{ch}(q / 2)}=\frac{2 \operatorname{sh}^{2}(\omega / 2)}{\operatorname{ch}(q / 2)} .
$$

Теорема доказана.

ЗАмЕчАНиЕ. Переходя в соотношении (6) к пределу при $q \rightarrow 0$, получаем утверждение теоремы 1.

Обратимся теперь к проинтегрированным процессам. В работах [2] и [5] проинтегрированный (однократно) процесс Боголюбова играет важную роль при установлении связей между мерами Боголюбова и Винера. Обозначим через $\xi_{n}^{\left[\alpha_{1}, \ldots, \alpha_{n}\right]}(t)$, $t \in[0,1], n$-кратно проинтегрированный процесс:

$$
\xi_{n}^{\left[\alpha_{1}, \ldots, \alpha_{n}\right]}(t)=(-1)^{\alpha_{1}+\cdots+\alpha_{n}} \int_{\alpha_{n}}^{t} \cdots \int_{\alpha_{1}}^{t_{1}} \xi(s) d s d t_{1} \ldots
$$

(каждый индекс $\alpha_{j}$ равен 0 либо 1). Эта общая форма многократно проинтегрированного процесса была впервые предложена в статье [22].

Проинтегрированные гауссовские процессы интенсивно изучаются в последние годы в связи с задачами проверки гипотез [23], непараметрического оценивания [24], изучением решений невязкого уравнения Бюргерса со случайными начальными данными [25], [26]; они также используются в метрологии как модели для погрешностей атомных часов с переменной точностью и синхронизацией [27].

В приведенной ниже теореме 3 вычислена точная асимптотика сразу для многократно проинтегрированного процесса Боголюбова. Следуя работе [15], введем при натуральном $\ell$ обозначения

$$
z_{\ell}=\exp \left(\frac{i \pi}{\ell}\right), \quad \mathcal{D}_{\ell}=\frac{2 \ell-1}{2 \ell \sin (\pi / 2 \ell)}, \quad \varepsilon_{\ell}=\left(\varepsilon \sqrt{2 \ell \sin \frac{\pi}{2 \ell}}\right)^{1 / 2 \ell-1},
$$

а при $j=1, \ldots, n$ - обозначения

$$
k_{j}=\left\{\begin{array}{ll}
n-j, & \text { если } \quad \alpha_{j}=0, \\
n+1+j, & \text { если } \quad \alpha_{j}=1,
\end{array} \quad k_{j}^{\prime}=2 n+1-k_{j} .\right.
$$

Через $V(\ldots)$ обозначим определитель Вандермонда. 
Теорема 3. При $\varepsilon \rightarrow 0$ верно соотношение

$$
\mathrm{P}\left\{\left\|\xi_{n}^{\left[\alpha_{1}, \ldots, \alpha_{n}\right]}\right\| \leqslant \varepsilon\right\} \sim \frac{2^{n+2}(n+1)^{n+1} \operatorname{sh}(\omega / 2)}{M^{1 / 2}} \frac{\varepsilon_{n+1}}{\sqrt{\pi \mathcal{D}_{n+1}}} \exp \left(-\frac{\mathcal{D}_{n+1}}{2 \varepsilon_{n+1}^{2}}\right),
$$

¿де

$$
M=\left|V\left(z^{k_{1}}, \ldots, z^{k_{n}}\right)\right|^{2}\left(\prod_{j=1}^{n}\left|1+z^{k_{j}}\right|^{2}+\prod_{j=1}^{n}\left|1+z^{k_{j}^{\prime}}\right|^{2}\right), \quad z=z_{n+1} .
$$

ДокАЗАТЕЛЬСтво. Из формулы (3.7) статьи [5] и теоремы 2.1 работы [13] следует, что собственные числа ковариации суть $\lambda_{k}=\mu_{k}^{-1}$, где $\mu_{k}$ - собственные числа краевой задачи

$$
\begin{gathered}
(-1)^{n+1}\left(u^{(2 n+2)}-\omega^{2} u^{(2 n)}\right)=\mu u \quad \text { на } \quad[0,1], \\
u^{(n-j)}\left(\alpha_{j}\right)=0, \quad j=1, \ldots, n, \\
u^{(n)}(0)=u^{(n)}(1), \quad u^{(n+1)}(0)=u^{(n+1)}(1), \\
\left(u^{(n+j+1)}-\omega^{2} u^{(n+j-1)}\right)\left(1-\alpha_{j}\right)=0, \quad j=1, \ldots, n .
\end{gathered}
$$

С учетом теоремы 1.2 из работы [16] нам достаточно показать, что

$$
C_{\text {dist }}^{2} \equiv \prod_{k=1}^{\infty} \frac{\mu_{k}}{(\pi(k-1 / 2))^{2 n+2}}=\frac{2^{n+2}(n+1)^{2 n+1} \operatorname{sh}^{2}(\omega / 2)}{M} .
$$

Обозначим через $\pm \zeta_{j}, j=0, \ldots, n$, корни уравнения

$$
\zeta^{2 n+2}+\omega^{2} \zeta^{2 n}=\mu .
$$

Будем считать, что $\zeta_{0}$ - тот корень уравнения, который положителен при положительных $\mu$. Тогда мы можем рассматривать $\zeta_{j}, j=1, \ldots, n$, как функции от $\zeta=\zeta_{0}$. Когда все корни уравнения (10) различны, функции $e^{ \pm i \zeta_{j} x}$ образуют фундаментальную систему. Подставляя их в граничные условия, получаем, что если $\mu-$ собственное значение задачи (9), то корни уравнения (10) являются нулями функции

$$
F(\zeta)=\operatorname{det}\left(A_{0}^{+}(\zeta), \ldots, A_{n}^{+}(\zeta), A_{0}^{-}(\zeta), \ldots, A_{n}^{-}(\zeta)\right)
$$

где

$$
A_{j}^{ \pm}(\zeta)=\left(\begin{array}{c}
\left( \pm i \zeta_{j}\right)^{n-1} e^{ \pm i \alpha_{1} \zeta_{j}} \\
\left( \pm i \zeta_{j}\right)^{n-2} e^{ \pm i \alpha_{2} \zeta_{j}} \\
\vdots \\
e^{ \pm i \alpha_{n} \zeta_{j}} \\
\left( \pm i \zeta_{j}\right)^{n}\left(1-e^{ \pm i \zeta_{j}}\right) \\
\left( \pm i \zeta_{j}\right)^{n+1}\left(1-e^{ \pm i \zeta_{j}}\right) \\
\left( \pm i \zeta_{j}\right)^{n}\left(\left(i \zeta_{j}\right)^{2}-\omega^{2}\right) e^{ \pm i\left(1-\alpha_{1}\right) \zeta_{j}} \\
\left( \pm i \zeta_{j}\right)^{n+1}\left(\left(i \zeta_{j}\right)^{2}-\omega^{2}\right) e^{ \pm i\left(1-\alpha_{2}\right) \zeta_{j}} \\
\vdots \\
\left( \pm i \zeta_{j}\right)^{2 n-1}\left(\left(i \zeta_{j}\right)^{2}-\omega^{2}\right) e^{ \pm i\left(1-\alpha_{n}\right) \zeta_{j}}
\end{array}\right) .
$$


Если же два корня уравнения (10) совпадают, то функция $F(\zeta)$ также обращается в нуль. Чтобы отбросить эти "лишние" нули, рассмотрим функцию

$$
\widetilde{F}(\zeta)=\frac{F(\zeta)}{V\left(\zeta_{0}, \ldots, \zeta_{n},-\zeta_{0}, \ldots,-\zeta_{n}\right)} .
$$

Теперь $\mu$ является собственным значением краевой задачи (9) тогда и только тогда, когда корни уравнения (10) являются нулями функции $\widetilde{F}(\zeta)$.

Заметим, что $\zeta_{j}=z^{j} \zeta+O\left(|\zeta|^{-1}\right), j=1, \ldots, n$, при $|\zeta| \rightarrow \infty$ Тогда при $|\zeta| \rightarrow \infty$

$$
\begin{aligned}
& \left|V\left(\zeta_{0}, \ldots, \zeta_{n},-\zeta_{0}, \ldots,-\zeta_{n}\right)\right| \sim \\
& \quad \sim|\zeta|^{(n+1)(2 n+1)}\left|V\left(1, z, \ldots, z^{2 n+1}\right)\right|=(2 n+2)^{n+1}|\zeta|^{(n+1)(2 n+1)} .
\end{aligned}
$$

Действуя по аналогии с рассуждениями из теоремы 2 в $§ 4$ книги [28], при $|\zeta| \rightarrow \infty$ и $|\arg \zeta| \leqslant \pi /(2 n+2)$ получаем

$$
F(\zeta)=\zeta^{(n+1)(2 n+1)} e^{-i z \zeta-i z^{2} \zeta-\cdots-i z^{n} \zeta}\left(\Phi(\zeta)+O\left(|\zeta|^{-1}\right)\right),
$$

где $\Phi(\zeta)=\operatorname{det}\left(\Phi_{1}(\zeta), \Phi_{2}(\zeta)\right)$,

$$
\begin{aligned}
& \Phi_{1}(\zeta)=\left(\begin{array}{cccc}
i^{n-1} e^{i \alpha_{1} \zeta} & (i z)^{n-1}\left(1-\alpha_{1}\right) & \ldots & \left(i z^{n}\right)^{n-1}\left(1-\alpha_{1}\right) \\
i^{n-2} e^{i \alpha_{2} \zeta} & (i z)^{n-2}\left(1-\alpha_{2}\right) & \ldots & \left(i z^{n}\right)^{n-2}\left(1-\alpha_{2}\right) \\
\vdots & \vdots & \ddots & \vdots \\
e^{i \alpha_{n} \zeta} & 1-\alpha_{n} & \ldots & 1-\alpha_{n} \\
i^{n}\left(1-e^{i \zeta}\right) & (i z)^{n} & \ldots & \left(i z^{n}\right)^{n} \\
i^{n+1}\left(1-e^{i \zeta}\right) & (i z)^{n+1} & \ldots & \left(i z^{n}\right)^{n+1} \\
i^{n+2} e^{i\left(1-\alpha_{1}\right) \zeta} & (i z)^{n+2} \alpha_{1} & \ldots & \left(i z^{n}\right)^{n+2} \alpha_{1} \\
i^{n+3} e^{i\left(1-\alpha_{2}\right) \zeta} & (i z)^{n+3} \alpha_{2} & \ldots & \left(i z^{n}\right)^{n+3} \alpha_{2} \\
\vdots & \vdots & \ddots & \vdots \\
i^{2 n+1} e^{i\left(1-\alpha_{n}\right) \zeta} & (i z)^{2 n+1} \alpha_{n} & \ldots & \left(i z^{n}\right)^{2 n+1} \alpha_{n}
\end{array}\right), \\
& \Phi_{2}(\zeta)=\left(\begin{array}{cccc}
(-i)^{n-1} e^{-i \alpha_{1} \zeta} & (-i z)^{n-1} \alpha_{1} & \ldots & \left(-i z^{n}\right)^{n-1} \alpha_{1} \\
(-i)^{n-2} e^{-i \alpha_{2} \zeta} & (-i z)^{n-2} \alpha_{2} & \ldots & \left(-i z^{n}\right)^{n-2} \alpha_{2} \\
\vdots & \vdots & \ddots & \vdots \\
e^{-i \alpha_{n} \zeta} & \alpha_{n} & \ldots & \alpha_{n} \\
(-i)^{n}\left(1-e^{-i \zeta}\right) & -(-i z)^{n} & \ldots & -\left(-i z^{n}\right)^{n} \\
(-i)^{n+1}\left(1-e^{-i \zeta}\right) & -(-i z)^{n+1} & \ldots & -\left(-i z^{n}\right)^{n+1} \\
(-i)^{n+2} e^{-i\left(1-\alpha_{1}\right) \zeta} & (-i z)^{n+2}\left(1-\alpha_{1}\right) & \ldots & \left(-i z^{n}\right)^{n+2}\left(1-\alpha_{1}\right) \\
(-i)^{n+3} e^{-i\left(1-\alpha_{2}\right) \zeta} & (-i z)^{n+3}\left(1-\alpha_{2}\right) & \ldots & \left(-i z^{n}\right)^{n+3}\left(1-\alpha_{2}\right) \\
\vdots & \vdots & \ddots & \vdots \\
(-i)^{2 n+1} e^{-i\left(1-\alpha_{n}\right) \zeta} & (-i z)^{2 n+1}\left(1-\alpha_{n}\right) & \ldots & \left(-i z^{n}\right)^{2 n+1}\left(1-\alpha_{n}\right)
\end{array}\right) .
\end{aligned}
$$


Учитывая обозначения и переставляя, если это необходимо, строки, получаем, что $\Phi(\zeta)=(-1)^{\alpha_{1}+\cdots+\alpha_{n}} \operatorname{det}\left(\Phi_{3}(\zeta), \Phi_{4}(\zeta)\right)$, где

$$
\begin{aligned}
& \Phi_{3}(\zeta)=\left(\begin{array}{cccc}
i^{k_{1}} & (i z)^{k_{1}} & \cdots & \left(i z^{n}\right)^{k_{1}} \\
i^{k_{2}} & (i z)^{k_{2}} & \cdots & \left(i z^{n}\right)^{k_{2}} \\
\vdots & \vdots & \ddots & \vdots \\
i^{k_{n}} & (i z)^{k_{n}} & \cdots & \left(i z^{n}\right)^{k_{n}} \\
i^{n}\left(1-e^{i \zeta}\right) & (i z)^{n} & \cdots & \left(i z^{n}\right)^{n} \\
i^{n+1}\left(1-e^{i \zeta}\right) & (i z)^{n+1} & \cdots & \left(i z^{n}\right)^{n+1} \\
i^{k_{1}^{\prime}} e^{i \zeta} & 0 & \cdots & 0 \\
i^{k_{2}^{\prime}} e^{i \zeta} & 0 & \cdots & 0 \\
\vdots & \vdots & \ddots & \vdots \\
i^{k_{n}^{\prime}} e^{i \zeta} & 0 & \cdots & 0
\end{array}\right) \\
& \Phi_{4}(\zeta)=\left(\begin{array}{cccc}
(-i)^{k_{1}} & 0 & \cdots & 0 \\
(-i)^{k_{2}} & 0 & \cdots & 0 \\
\vdots & \vdots & \ddots & \vdots \\
(-i)^{k_{n}} & 0 & \ldots & 0 \\
(-i)^{n}\left(1-e^{-i \zeta}\right) & -(-i z)^{n} & \ldots & -\left(-i z^{n}\right)^{n} \\
(-i)^{n+1}\left(1-e^{-i \zeta}\right) & -(-i z)^{n+1} & \ldots & -\left(-i z^{n}\right)^{n+1} \\
(-i)^{k_{1}^{\prime}} e^{-i \zeta} & (-i z)^{k_{1}^{\prime}} & \ldots & \left(-i z^{n}\right)^{k_{1}^{\prime}} \\
(-i)^{k_{2}^{\prime}} e^{-i \zeta} & (-i z)^{k_{2}^{\prime}} & \cdots & \left(-i z^{n}\right)^{k_{2}^{\prime}} \\
\vdots & \vdots & \ddots & \vdots \\
(-i)^{k_{n}^{\prime}} e^{-i \zeta} & (-i z)^{k_{n}^{\prime}} & \cdots & \left(-i z^{n}\right)^{k_{n}^{\prime}}
\end{array}\right) .
\end{aligned}
$$

В результате непосредственных вычислений имеем $|\Phi(\zeta)|=M\left|e^{i \zeta}+e^{-i \zeta}+R\right|$, где

$$
\begin{aligned}
M=\mid & V\left(z^{k_{1}}, \ldots, z^{k_{n}}, z^{n}\right) V\left(z^{n+1}, z^{k_{1}^{\prime}}, \ldots, z^{k_{n}^{\prime}}\right)+ \\
& +V\left(z^{k_{1}}, \ldots, z^{k_{n}}, z^{n+1}\right) V\left(z^{n}, z^{k_{1}^{\prime}}, \ldots, z^{k_{n}^{\prime}}\right) \mid,
\end{aligned}
$$

a $R$ - константа, значение которой для дальнейших вычислений несущественно. Заметим, что выражение для $M$ можно переписать так:

$$
M=\left|V\left(z^{k_{1}}, \ldots, z^{k_{n}}\right)\right|^{2}\left(\prod_{j=1}^{n}\left|1+z^{k_{j}}\right|^{2}+\prod_{j=1}^{n}\left|1+z^{k_{j}^{\prime}}\right|^{2}\right) .
$$

Рассмотрим функцию [15]

$$
\Psi_{\delta}(\zeta)=\psi_{\delta}(\zeta) \psi_{\delta}(z \zeta) \ldots \psi_{\delta}\left(z^{n} \zeta\right)
$$

где

$$
\psi_{\delta}(\zeta)=\frac{\Gamma^{2}(1+\delta)}{\Gamma(1+\delta+\zeta / \pi) \Gamma(1+\delta-\zeta / \pi)}=\prod_{k=1}^{\infty}\left(1-\frac{\zeta^{2}}{(\pi(k+\delta))^{2}}\right)
$$


Как было показано в работе [15], при $\zeta \rightarrow \infty,|\arg \zeta| \leqslant \phi_{0}<\pi$,

$$
\psi_{\delta}(\zeta) \sim \Gamma^{2}(1+\delta) \pi^{2 \delta} \zeta^{-2 \delta-1} \cos \left(\zeta-\pi\left(\delta+\frac{1}{2}\right)\right),
$$

причем сходимость равномерная. Полагая $\delta=-1 / 2$, получаем, что

$$
\frac{|\widetilde{F}(\zeta)|}{\left|\Psi_{\delta}(\zeta)\right|} \rightarrow \frac{2^{n+1} M}{(2 n+2)^{2 n+1}}
$$

если $\zeta \rightarrow \infty, \arg \zeta \neq \pi j /(2 n+2), j \in \mathbb{Z}$.

Применяя теорему Иенсена к функциям $\widetilde{F}(\zeta)$ и $\Psi_{\delta}(\zeta)$, с учетом теоремы Лебега о мажорируемой сходимости получаем

$$
C_{\text {dist }}^{2}=\frac{(2 n+2)^{2 n+1}|\widetilde{F}(0)|}{2^{n+1} M} .
$$

Перенумеровав $\zeta_{j}, j=1, \ldots, n$, мы можем считать, что при $\zeta \rightarrow 0$

$$
\zeta_{j}=z_{n}^{j} \zeta(1+O(|\zeta|)), \quad j=1, \ldots, n-1 ; \quad \zeta_{n} \rightarrow \omega i .
$$

Тогда при $\zeta \rightarrow 0$

$$
\begin{aligned}
& \left|V\left(\zeta_{0}, \ldots, \zeta_{n},-\zeta_{0}, \ldots,-\zeta_{n}\right)\right| \sim \\
& \quad \sim 2 \omega^{4 n+1}|\zeta|^{n(2 n-1)}\left|V\left(1, z_{n}, \ldots, z_{n}^{2 n-1}\right)\right|=2(2 n)^{n} \omega^{4 n+1}|\zeta|^{n(2 n-1)}
\end{aligned}
$$

и $F(\zeta) \sim \pm \zeta^{n(2 n-1)} \operatorname{det}\left(\Phi_{5}(\zeta), \Phi_{6}(\zeta)\right)$, где

$$
\begin{aligned}
& \Phi_{5}(\zeta)=\left(\begin{array}{cccc}
i^{n-1} & \ldots & \left(i z_{n}^{n-1}\right)^{n-1} & \left(-\omega \zeta^{-1}\right)^{n-1} e^{-\alpha_{1} \omega} \\
i^{n-2} & \ldots & \left(i z_{n}^{n-1}\right)^{n-2} & \left(-\omega \zeta^{-1}\right)^{n-2} e^{-\alpha_{2} \omega} \\
\vdots & \ddots & \vdots & \vdots \\
1 & \ldots & 1 & e^{-\alpha_{n} \omega} \\
(i \zeta)^{n} & \ldots & \left(i z_{n}^{n-1} \zeta\right)^{n} & (-\omega)^{n}\left(1-e^{-\omega}\right) \\
(i \zeta)^{n+1} & \ldots & \left(i z_{n}^{n-1} \zeta\right)^{n+1} & (-\omega)^{n+1}\left(1-e^{-\omega}\right) \\
-\omega^{2} i^{n} & \ldots & -\omega^{2}\left(i z_{n}^{n-1}\right)^{n} & 0 \\
-\omega^{2} i^{n+1} & \ldots & -\omega^{2}\left(i z_{n}^{n-1}\right)^{n+1} & 0 \\
\vdots & \ddots & \vdots & \vdots \\
-\omega^{2} i^{2 n-1} & \ldots & -\omega^{2}\left(i z_{n}^{n-1}\right)^{2 n-1} & 0
\end{array}\right) \\
& \Phi_{6}(\zeta)=\left(\begin{array}{cccc}
(-i)^{n-1} & \cdots & \left(-i z_{n}^{n-1}\right)^{n-1} & \left(\omega \zeta^{-1}\right)^{n-1} e^{\alpha_{1} \omega} \\
(-i)^{n-2} & \cdots & \left(-i z_{n}^{n-1}\right)^{n-2} & \left(\omega \zeta^{-1}\right)^{n-2} e^{\alpha_{2} \omega} \\
\vdots & \ddots & \vdots & \vdots \\
1 & \ldots & 1 & e^{\alpha_{n} \omega} \\
(-i \zeta)^{n} & \cdots & \left(-i z_{n}^{n-1} \zeta\right)^{n} & \omega^{n}\left(1-e^{\omega}\right) \\
(-i \zeta)^{n+1} & \ldots & \left(-i z_{n}^{n-1} \zeta\right)^{n+1} & \omega^{n+1}\left(1-e^{\omega}\right) \\
-\omega^{2}(-i)^{n} & \ldots & -\omega^{2}\left(-i z_{n}^{n-1}\right)^{n} & 0 \\
-\omega^{2}(-i)^{n+1} & \ldots & -\omega^{2}\left(-i z_{n}^{n-1}\right)^{n+1} & 0 \\
\vdots & \ddots & \vdots & \vdots \\
-\omega^{2}(-i)^{2 n-1} & \ldots & -\omega^{2}\left(-i z_{n}^{n-1}\right)^{2 n-1} & 0
\end{array}\right)
\end{aligned}
$$


Разлагая по $(n+1)$-му и $(2 n+2)$-му столбцам, получаем

$$
\begin{aligned}
&|F(\zeta)| \sim|\zeta|^{n(2 n-1)}\left|V\left(i, i z_{n}, \ldots, i z_{n}^{2 n-1}\right)\right| \omega^{2 n} \times \\
& \quad \times\left|\operatorname{det}\left(\begin{array}{cc}
(-\omega)^{n}\left(1-e^{-\omega}\right) & \omega^{n}\left(1-e^{\omega}\right) \\
(-\omega)^{n+1}\left(1-e^{-\omega}\right) & \omega^{n+1}\left(1-e^{\omega}\right)
\end{array}\right)\right|= \\
&=8(2 n)^{n} \omega^{4 n+1} \operatorname{sh}^{2}\left(\frac{\omega}{2}\right)|\zeta|^{n(2 n-1)} .
\end{aligned}
$$

Следовательно, $|\widetilde{F}(0)|=4 \operatorname{sh}^{2}(\omega / 2)$ и

$$
C_{\mathrm{dist}}^{2}=\frac{2^{n+2}(n+1)^{2 n+1} \operatorname{sh}^{2}(\omega / 2)}{M} .
$$

Теорема доказана.

ЗАмечАниЕ. Полагая в соотношении (8) $n=0$, получаем утверждение теоремы 1.

Благодарности. Автор признателен А. И. Назарову и Я. Ю. Никитину за внимание к работе и рецензенту за замечания, способствовавшие улучшению статьи. Работа выполнена при частичной финансовой поддержке РФФИ (грант № 10-01-00154) и Программы поддержки ведущих научных школ (грант НШ-4472.2010.1).

\section{Список литературы}

[1] Д. П. Санкович, ТМФ, 119:2 (1999), 345-352.

[2] Д. П. Санкович, ТМФ, 126:1 (2001), 149-163.

[3] Н. Н. Боголюбов, Докл. АН СССР, 99:2 (1954), 225-226.

[4] Д. П. Санкович, ТМФ, 127:1 (2001), 125-142.

[5] В. Р. Фаталов, ТМФ, 157:2 (2008), 286-308.

[6] W.V. Li, Q.-M. Shao, "Gaussian processes: inequalities, small ball probabilities and applications", Stochastic Processes: Theory and Methods, Handbook Statist., 19, eds. D. N. Shanbhag, C. R. Rao, North-Holland, Amsterdam, 2001, 533-597.

[7] M. A. Lifshits, Bibliography of small deviation probabilities. http://www.proba.jussieu.fr/pageperso/smalldev/biblio.pdf.

[8] F. Ferraty, P. Vieu, Nonparametric Functional Data Analysis, Springer Ser. Statist., Springer, New York, 2006.

[9] A. W. van der Vaart, J. H. van Zanten, Ann. Statist., 36:3 (2008), 1435-1463.

[10] F. Aurzada, И. А. Ибрагимов, М. А. Лифшиц, Н. J. van Zanden, ТВП, 53:4 (2008), 788-798.

[11] L. Beghin, Ya. Yu. Nikitin, E. Orsingher, "Exact small ball constants for some Gaussian processes under $L^{2}$-norm", Вероятность и статистика. 6, Зап. научн. сем. ПОМИ, 298, ПОМИ, СПб., 2003, 5-21.

[12] Я. Ю. Никитин, П. А. Харинский, “Точная асимптотика малых уклонений в $L_{2}$-норме для одного класса Гауссовских процессов”, Вероятность и статистика. 7, Зап. научн. сем. ПОМИ, 311, ПОМИ, СПб., 2004, 214-221.

[13] A. I. Nazarov, Ya. Yu. Nikitin, Probab. Theory Related Fields, 129:4 (2004), 469-494.

[14] А. И. Назаров, Р. С. Пусев, “Точная асимптотика малых уклонений в $L_{2}$-норме с весом для некоторых гауссовских процессов", Вероятность и статистика. 14-2, Зап. научн. сем. ПОМИ, 364, ПОМИ, СПб., 2009, 166-199. 
[15] А. И. Назаров, Проблемы матем. анализа, 26 (2003), 179-213.

[16] A. I. Nazarov, J. Theoret. Probab., 22:3 (2009), 640-665.

[17] R. J. Adler, An Introduction to Continuity, Extrema and Related Topics for General Gaussian Processes, IMS Lecture Notes - Monograph Ser., 12, Institute of Mathematical Statistics, Hayward, California, 1990.

[18] В. А. Зорич, Математический анализ, Часть 2, МЦНМО, М., 1998.

[19] Э. Камке, Справочник по обыкновенным дифференциальным уравнениям, Лань, СПб., 2003.

[20] И. С. Градштейн, И.М. Рыжик, Таблицы интегралов, сумм, рядов и произведений, Физматлит, М., 1971.

[21] Е. Титчмарш, Теория функиий, Наука, М., 1980.

[22] F. Gao, J. Hannig, F. Torcaso, Ann. Probab., 31:3 (2003), 1320-1337.

[23] A. Lachal, Math. Meth. Statist., 10 (2001), 73-104.

[24] P. Groeneboom, G. Jongbloed, J. A. Wellner, Ann. Statist., 29:6 (2001), 1620-1652.

[25] Я. Г. Синай, ТМФ, 90:3 (1992), 323-353.

[26] G. Molchan, A. Khokhlov, J. Stat. Phys., 114:3-4 (2004), 923-946.

[27] L. Galleani, L. Sacerdote, P. Tavella, C. Zucca, Metrologia, 40:3 (2003), S257-S264.

[28] М. А. Наймарк, Линейные дифференциальные операторы, Наука, М., 1969.

Поступила в редакцию 24.03.2010, после доработки 26.04.2010 